\title{
Larval development of Hypsophrys nicaraguensis (Pisces: Cichlidae) under laboratory conditions
}

\author{
Alex Molina Arias \\ Instituto Costarricense de Electricidad, Estación Piscícola de la Unidad de Cuenca del Río Peñas Blancas; \\ molalx@yahoo.com,amolinaa@ice.go.cr
}

Received 03-IX-2010. C Corrected 04-II-2011. Accepted 07-III-2011.

\begin{abstract}
The cichlid Hypsophrys nicaraguensis is a popular fish known as butterfly, and despite its widespread use as pets, little is known about its reproductive biology. In order to contribute to this knowledge, the study describes the relevant larval development characteristics, from adult and larval cultures in captivity. Every $12 \mathrm{~h}$, samples of larvae were collected and observed under the microscope for larval stage development, and every $24 \mathrm{~h}$ morphometric measurements were taken. Observations showed that at $120 \mathrm{~h}$, some larvae had swimming activity and the pectoral fins development was visible; at 144h, the dorsal fin appear and all larvae started food intake; at $168 \mathrm{~h}$, the formation of anal fins begins, small rudiments of pelvic fins emerge, the separation of caudal fin from anal and dorsal fins starts, and the yolk sac is reabsorbed almost completely; at $288 \mathrm{~h}$, the pelvic fins starts to form; at $432 \mathrm{~h}$, the rays and spines of dorsal and anal fins can be distinguished, both the anal and the dorsal fins have the same number of spines and rays as in adults. After $480 \mathrm{~h}$ larvae have the first scales, ending the larval stages and starting the transformation to fingerlings. Larvae were successfully fed with commercial diet. Rev. Biol. Trop. 59 (4): 1679-1684. Epub 2011 December 01.
\end{abstract}

Key words: Hypsophrys nicaraguensis, cichlidae, butterfly cichlid, larvae, larval development.

Hypsophrys nicaraguensis (Agassiz 1859) se conoce comúnmente como "moga amarilla" o "butterfly", se distingue por su gran mancha negra en medio costado, atravesado o no por una estría lateral, su cola recortada y el perfil de la cabeza curvo con la boca en posición inferior. El color general de la cabeza es azul verde grisáceo y el cuerpo es dorado a cobrizo en individuos no activos sexualmente. El dimorfismo sexual es claro, los machos son más grandes que las hembras y a menudo con giba nucal, el color dorado del cuerpo es más intenso a un anaranjado y las escamas están bordeadas en pardo, dando un efecto reticulado. En estos machos adultos, la mancha y estría lateral tienden a desaparecer, pero las aletas impares anaranjadas están cubiertas de pintas negras. La hembra en cambio es más pequeña y tiene la mancha y estría lateral muy evidentes y las aletas impares carecen de manchas y la dorsal es verde azulada. A diferencia de la mayoría de las otras mojarras, esta especie no adhiere los huevecillos a rocas o troncos, sino que los deposita en depresiones en el barro del fondo. Alcanzan 165mm de largo. Se distribuye en la Vertiente Atlántica desde el lago de Nicaragua y el sistema del río San Juan y tributarios hasta la cuenca del río Matina en la Vertiente Atlántica de Costa Rica. Habita ríos y riachuelos con poca a moderada corriente, en aguas entre 5 y $200 \mathrm{~m}$ de altura y temperaturas entre $23^{\circ} \mathrm{C}$ a $36^{\circ} \mathrm{C}$ (Bussing 2002).

$H$. nicaraguensis es una especie popular entre los amantes de la acuariofilia, existe gran cantidad de sitios en Internet que describen sus requerimientos físico-químicos y comportamiento para mantenimiento en cautiverio (Fariñas 2009, Animal World 2009) y solamente se 
han realizado estudios a nivel de sistemática y de inventarios biológicos (Coleman 1999, Bussing 2002, Chakrabarty \& Sparks 2007).

El presente trabajo tiene el propósito de contribuir al conocimiento científico de la biología reproductiva de $H$. nicaraguensis, aportando información desde sus etapas iniciales de desarrollo a partir del desove, hasta su transformación como alevín.

\section{MATERIALES Y MÉTODOS}

Primeramente se observaron huevos y larvas de $H$. nicaraguensis provenientes de tres desoves de reproductores recolectados en el río Peñas Blancas, San Ramón, Alajuela, Costa Rica $\left(10^{\circ} 29^{\prime} 002^{\prime \prime} \mathrm{N}-84^{\circ} 29^{\prime} 421^{\prime \prime} \mathrm{W}\right)$ y se mantuvieron durante 14 meses bajo condiciones de laboratorio, en la Estación Piscícola Peñas Blancas del Instituto Costarricense de Electricidad, con un flujo de agua continuo y una temperatura de $26 \pm 1^{\circ} \mathrm{C}$.

Seguidamente se observó el tiempo de desarrollo y las medidas promedio del largo y ancho de los huevos incubados por los padres. Las larvas obtenidas de la reproducción natural, se extrajeron inmediatamente después de la eclosión y fueron criadas en acuarios de $25 \mathrm{~L}$ a $27.0 \pm 0.5^{\circ} \mathrm{C}$, con fotoperíodo de $12-12 \mathrm{~h}$ y $\mathrm{pH}$ de $7.5 \pm 0.5$ y se alimentaron ad libitum, se alternó artemia (Artemia franciscana) con alimento para tilapia ( $30 \%$ proteína) pulverizado, cuatro veces al día.

Post eclosión cada doce horas por un periodo de 25 días, se fijó una muestra en formalina al $5 \%$ y luego de $24 \mathrm{~h}$ se transfirió a alcohol al 50\% para su posterior observación. Las mediciones morfométricas se realizaron cada $24 \mathrm{~h}$, bajo estereoscopio y microscopio con una reglilla micrométrica con una precisión de $0.01 \mathrm{~mm}$ y el análisis del desarrollo larval, de acuerdo con Molina (2008). El criterio para determinar la aparición de las aletas se basó en la formación inicial de las yemas o protuberancias de las aletas pares (pélvicas y pectorales) y para las impares (dorsal, anal y caudal) se utilizó la aparición por primera vez de los radios y/o espinas (Molina 2008).
Las muestras preservadas se encuentran depositadas en la Estación Piscícola Peñas Blancas del Instituto Costarricense de Electricidad, San Ramón, Alajuela, Costa Rica, con el número de catálogo EP-PBL-09-02.

\section{RESULTADOS}

H. nicaraguensis presenta un comportamiento parental de proteger los huevos y larvas, únicamente por parte de la madre. Los huevos no son adheridos al sustrato, son depositados directamente en el fondo del estanque, en un lugar protegido, para no ser arrastrados por corrientes o movimientos del agua. Los huevos eclosionan entre las $78 \mathrm{~h}$ y $80 \mathrm{~h}$, son ovalados con una longitud de $2.00 \pm 0.05 \mathrm{~mm}$ y de ancho $1.55 \pm 0.05 \mathrm{~mm}$.

Fase de Protolarva: Esta fase se inicia con la eclosión de la larva. El embrión recién eclosionado mide en promedio $5.28 \pm 0.04 \mathrm{~mm}$ de longitud total $(\mathrm{Lt})$, el saco vitelino es ovalado con un tamaño de $0.68 \pm 0.02 \mathrm{~mm}$ (Cuadro 1 ), solamente en la zona caudal se observan movimientos, mientras que la cabeza se mantiene reposando en el fondo. La cabeza y los ojos se observan claramente, no es evidente aún la boca ni las aletas pectorales, además tienen la particularidad de que, a pesar que emergen con las glándulas cefálicas, la secreción de éstas no es suficiente para adherirlas fijamente al sustrato (Fig. 1A).

A las $24 \mathrm{~h}$ post eclosión, los ojos están ligeramente desarrollados con poca pigmentación y se observan los primordios de las aletas pectorales (Fig. 1B). A partir de las $48 \mathrm{~h}$ se puede ver la abertura bucal. Las seis glándulas de adhesión de la cabeza se reducen considerablemente de tamaño (Fig. 1C). A las $72 \mathrm{~h}$ se observan las branquias, las cuales no son cubiertas aún por el opérculo (Fig. 1D). A las 96h se comienzan a forman los primeros radios de la aleta caudal, el saco vitelino cambia de una forma ovalada hacia una forma circular y el opérculo cubre completamente las branquias (Fig. 1E). A las $120 \mathrm{~h}$ algunas larvas comienzan a nadar erráticamente, y se comienzan a formar algunos 


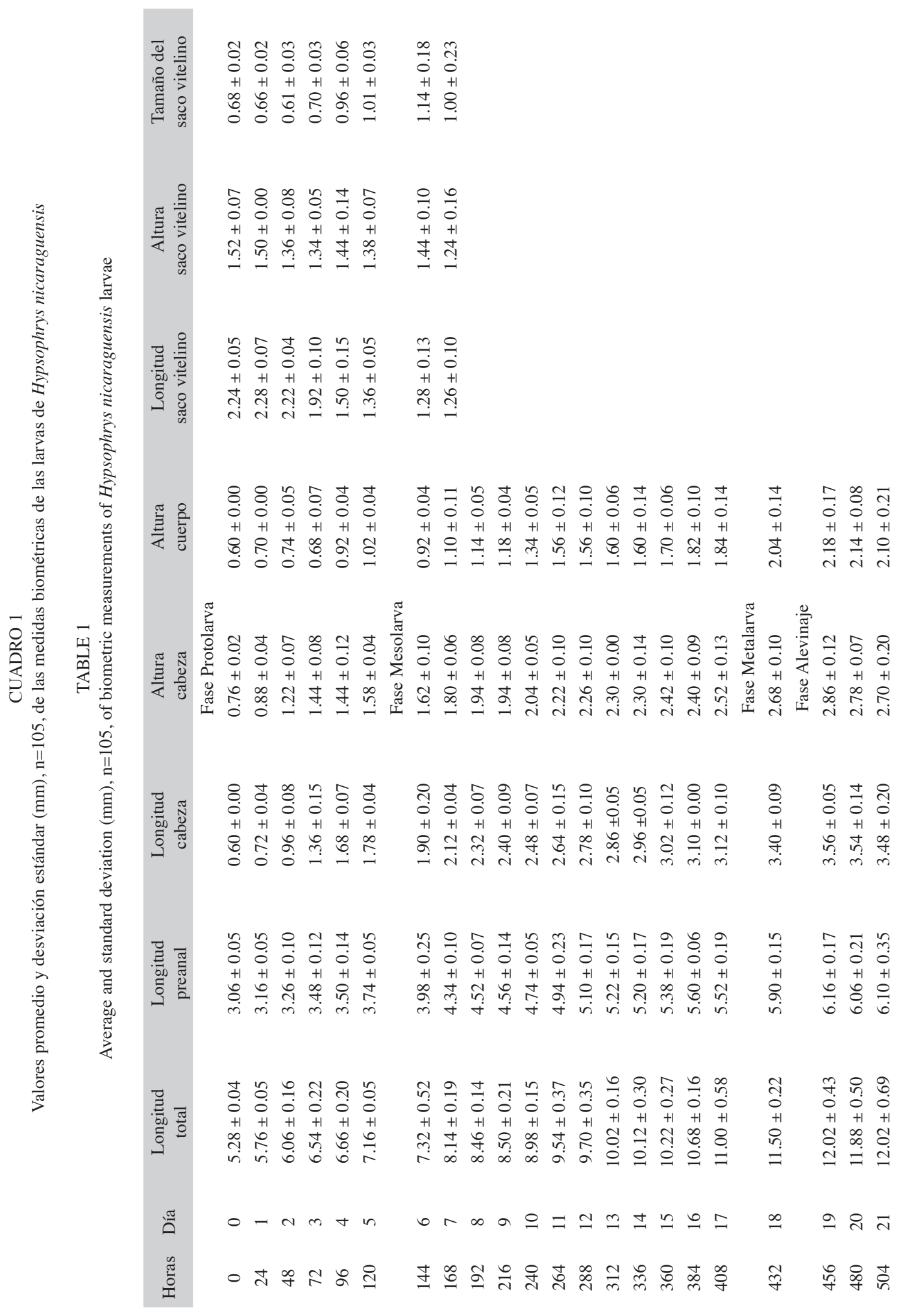




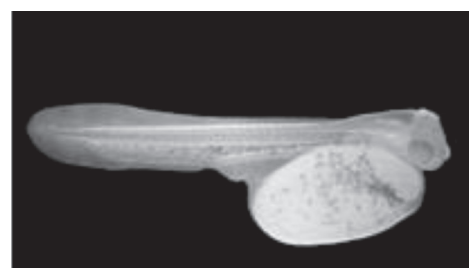

A

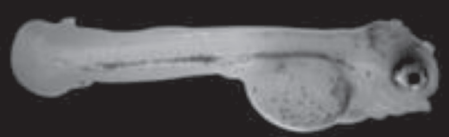

D

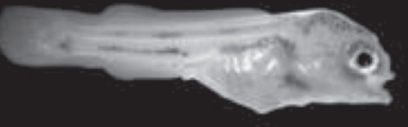

G

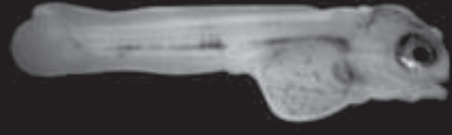

E

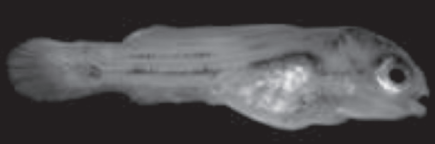

H

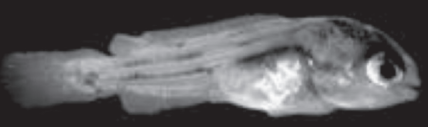

K

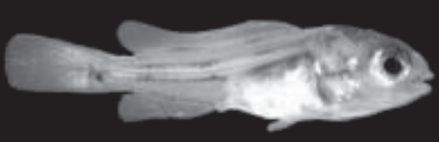

$\mathbf{N}$

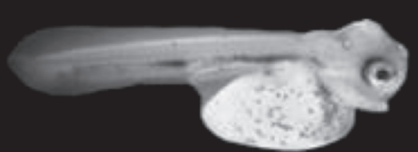

C

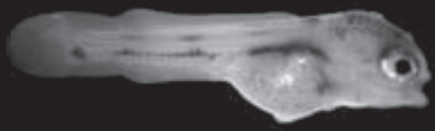

F

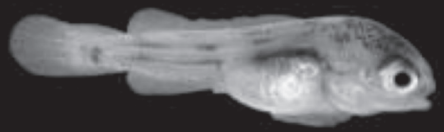

I

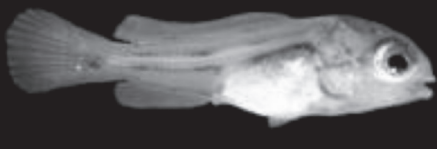

L

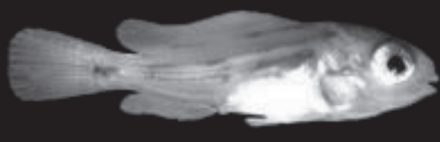

O

Fig. 1. Desarrollo larval de Hypsophrys nicaraguensis: (A) 0h; (B) 24h; (C) 48h; (D) 72h; (E) 96h; (F) 120h; (G) 144h; (H) 168h; (I) 192h; (J) 216h; (K) 240h; (L) 288h; (M) 312h; (N) 336h; (O) 480h.

Fig. 1. Larval development of Hypsophrys nicaraguensis: (A) 0h; (B) 24h; (C) 48h; (D) 72h; (E) 96h; (F) 120h; (G) 144h; (H) 168h; (I) 192h; (J) 216h; (K) 240h; (L) 288h; (M) 312h; (N) 336h; (O) 480h. 
radios en las aletas pectorales (Fig. 1F). A las $144 \mathrm{~h}$ todas las larvas comienzan a alimentarse y se forman los primeros radios (sin segmentación) de la aleta dorsal (Fig. 1G).

Fase de Mesolarva: Esta etapa se inicia a las $168 \mathrm{~h}$ con la formación de los primeros radios de las aletas anal y dorsal. Luego comienzan a emerger los rudimentos de las aletas pélvicas, a manera de un par de pequeñas protuberancias. Posterioromente se inicia la separación las aletas dorsal y anal de la aleta caudal y el saco vitelino es reabsorbido casi completamente (Fig. 1H). A las 192h se observa crecimiento de las aletas pélvicas aunque aún no el desarrollo de los radios (Fig. 1I). A las $216 \mathrm{~h}$ la separación de la aleta caudal de las aletas dorsal y anal es clara, pero aún persiste el pliegue que recubre a estas últimas (Fig. 1J). Desde las $240 \mathrm{~h}$ (Fig. 1K) y hasta las $28 \mathrm{~h}$ no se observan cambios notables en estructuras externas, pero si en cuanto a crecimiento. A las $288 \mathrm{~h}$ se forman los primeros radios de las aletas pélvicas y algunos radios de la aleta dorsal comienzan a segmentarse (Fig. 1L).

A las 336h se observa el inicio de una segmentación difusa en algunos radios de la aleta anal (Fig. 1M).

Fase de Metalarva: Esta etapa comienza cuando se forman las aletas pélvicas y se ha completado el número común de espinas y radios en las aletas impares. Sin embargo, en este caso las aletas pélvicas ya se han formado durante la Fase de Mesolarva a las 288h. A las $432 \mathrm{~h}$ se distinguen radios de espinas en aletas impares y las aletas anal y dorsal alcanzan el mismo número de radios y espinas de un adulto. Además, se observan 8 espinas y 6 a 7 radios en la aleta anal y 19 espinas y 9 a 10 radios en la aleta dorsal (Fig. 1N).

Fase de Alevinaje: Entre las $456 \mathrm{~h}$ y las 480h surgen las primeras escamas en las larvas y se inicia así la etapa de alevín (Fig. 1O). Los alevines completamente formados tienen características distintivas tales como perfil de la cabeza curvo, poca cantidad de cromatóforos concentrados principalmente en la cabeza en la parte superior por detrás del ojo, una delgada franja a lo largo de la línea media del costado del cuerpo, otra línea a lo largo de la base de las aletas dorsal y anal y una concentración de cromatóforos formando una mancha conspicua en el pedúnculo caudal. No se observa concentración de cromatóforos en la zona media del cuerpo donde en adultos existe una gran mancha circular.

\section{DISCUSIÓN}

En cuanto a tiempos de desarrollo larval, se observa que $H$. nicaraguensis presenta un desarrollo embrionario de $78 \mathrm{~h}$ a $80 \mathrm{~h}$, el cual es un poco mayor que el de otras especies cultivadas bajo las mismas condiciones tales como Amphilophus rostratus (65h a 70h), Amphilophus citrinellus (70h a 74h) y Amphilophus alfari (68h a 70h) (Molina 2008, Molina 2009, Molina 2010).

El periodo en que las larvas consumen el saco vitelino y comienzan a alimentarse exógenamente se da a las $144 \mathrm{~h}$ y coincide aproximadamente con el de Cichlasoma nigrofasciatum, Cichlasoma managuense, Cichlasoma sp, Herotilapia multispinosa, Cichlasoma dimerus, A. rostratus, A. citrinellus, A. alfari y Cichlasoma dovii (Martínez \& Murillo 1987, Rodríguez et al. 1989, Murillo 1990, Murillo \& Cabrera 1993, Meijide \& Guerrero 2000, Molina 2008, Molina 2009, Molina 2010, obs. pers.), sin embargo a partir de la fase mesolarva se observa una divergencia de crecimiento (tiempos de desarrollo larval) entre algunas de las especies mencionadas, por ejemplo la fase mesolarva en $H$. nicaraguensis inicia a las $168 \mathrm{~h}$, sin embargo para A. citrinellus inicia a las $192 \mathrm{~h}$. H. nicaraguensis entra a la etapa de alevinaje entre las $456 \mathrm{~h}$ y $480 \mathrm{~h}$ al igual que $A$. rostratus (Molina 2008) pero distinto que $C$. sp y $A$. alfari las cuales inician a las 504h (Murillo 1990, Molina 2009), en tanto que A. citrinellus entra a esta etapa hasta las 744h (Molina 2010).

Una característica particular de $H$. nicaraguensis es que las larvas a pesar de eclosionar 
con glándulas en la cabeza para adherirse al sustrato, éstas parecen no ser funcionales, debido a que las larvas luego de la eclosión permanecen en el fondo agrupadas pero sin fijarse al sustrato, de tal manera que con una leve corriente pueden ser desagrupadas. Este aspecto las hace muy diferentes al resto de los cíclidos costarricenses, los cuales en su totalidad se adhieren al sustrato en las fases larvales.

\section{AGRADECIMIENTOS}

El autor agradece a Juan Rodríguez y Julio Gamboa por su valiosa colaboración en el mantenimiento y cuidado de los peces y al Instituto Costarricense de Electricidad de Costa Rica y su Unidad de Cuenca del río Peñas Blancas por el apoyo brindado a las investigaciones que se desarrollan en la Estación Piscícola.

\section{RESUMEN}

Hypsophrys nicaraguensis (Agassiz 1859) se conoce comúnmente como "moga amarilla" o "butterfly", se distingue por su gran mancha negra en medio costado, atravesado o no por una estría lateral, su cola recortada y el perfil de la cabeza curvo con la boca en posición inferior. Con el propósito de contribuir al conocimiento de la biología reproductiva de Hypsophrys nicaraguensis, se describen las características más relevantes del desarrollo larval. Cada $12 \mathrm{~h}$ se tomaron muestras de larvas cultivadas en cautiverio para observar la evolución del desarrollo larval y cada $24 \mathrm{~h}$ se realizó el registro de las medidas morfométricas. Entonces se observó que a las 120h algunas larvas pueden nadar y se comienzan a formar las aletas pectorales; a las $144 \mathrm{~h}$ inicia la formación de la aleta dorsal y todas las larvas empiezan a comer; a las $168 \mathrm{~h}$ se da la formación de la aleta anal, los rudimentos de las pélvicas se van formando, las aletas anal y dorsal se separan poco a poco de la aleta caudal y el saco vitelino es reabsorbido casi completamente; a las $288 \mathrm{~h}$ se observa el inicio de la formación de las aletas pélvicas; a las $432 \mathrm{~h}$ se distinguen radios de espinas en las aletas impares y las aletas anal y dorsal alcanzan el mismo número de radios y espinas que un adulto; después de las $480 \mathrm{~h}$ se forman las primeras escamas y concluye el desarrollo larval y da el inicio de la etapa de alevín.

Palabras clave: Hypsophrys nicaraguensis, cichlidae, cíclido mariposa, larva, desarrollo larval.

\section{REFERENCIAS}

Animal World. 2009. Nicaragua Cichlid. (Consultado: 01 abril 2009, http://animal-world.com/encyclo/fresh/ cichlid/nicarag.php).

Bussing, W. 2002. Peces de las aguas continentales de Costa Rica. Universidad de Costa Rica, San José, Costa Rica.

Chakrabarty, P. \& J. Sparks. 2007. Relationships of the New World cichlid genus Hypsophrys (Agassiz 1859) (Teleostei: Cichlidae) with diagnoses for the genus and its species. Zootaxa 1523: 59-64.

Coleman, R. 1999. Cichlids of the rio Puerto Viejo. Costa Rica. Cich. News Mag. 8: 6-12.

Fariñas, J.M. 2009. Hypsophrys nicaraguensis. (Consultado: 01 de abril 2009, http://atlas.drpez.org/ Hypsophrys_nicaraguensis).

Martínez, G. \& R. Murillo. 1987. Desarrollo larval de Cichlasoma nigrofasciatum (Günther), 1868 (Pisces: Cichlidae) en cultivos en laboratorio. Rev. Biol. Trop. 35: 113-119.

Meijide, F.J. \& G. Guerrero 2000. Embryonic and larval development of a substrate-brooding cichlid Cichlasoma dimerus (Heckel, 1840) under laboratory conditions. J. Zool. 252: 481-493.

Molina, A. 2008. Desarrollo larval de Amphilophus rostratus (Guill 1877) (Pisces:Cichlidae) cultivados en laboratorio. Brenesia 70: 47-52.

Molina, A. 2009. Desarrollo larval de Amphilophus citrinellus (Günter 1864) (Pisces Cichlidae) cultivados en laboratorio. Brenesia en prensa.

Molina, A. 2010. Desarrollo larval de Amphilophus alfari (Meek 1907) (Pisces Cichlidae) cultivados en laboratorio. Brenesia 73-74: 103-107.

Murillo, R. 1990. Desarrollo larval de Cichlasoma sp. (Pisces: Cichlidae) cultivados en laboratorio. Brenesia 33: 1-13.

Murillo, R. \& J. Cabrera. 1993. Desarrollo embrionario, larval y del alevín de Herotilapia multispinosa (Gunter 1868) (Pisces: Cichlidae), p. 11-17. In Actas del Simposio "Investigación Acuícola Acuacultura y Pesca en Centroamérica”. Heredia, Costa Rica.

Rodríguez, J., O. Alpirez \& R. Murillo. 1989. Desarrollo larval de Cichlasoma managuense (Pisces: Cichlidae) en condiciones de laboratorio. Brenesia 31: 11-20. 